\title{
带预载的钢丝网套补偿器振动疲劳损伤分析
}

\author{
杨 燕 ${ }^{1}$ 贺启林 $^{1}$ 朱海洋 $^{2}$ 司会柳 $^{1}$ 王 琦 $^{2}$ 任明法 $^{2}$ \\ (1. 北京宇航系统工程研究所 北京 100076; \\ 2. 大连理工大学工程力学系 大连 116024)
}

\begin{abstract}
摘要: 开展了基于有限元分析软件 ABAQUS 的钢丝网套补偿器在随机振动工况和正弦振动工况下的疲劳分析。首先采用梁 单元建立钢丝网套等效模型, 针对不同载荷工况构建不同的钢丝网套与波纹管间的相互作用关系, 从而建立能够考虑预载影 响的钢丝网套补偿器随机振动、正弦振动分析模型, 并开展支反力试验和模态测试对模型进行验证。基于该模型, 结合 Dirlik 方法及基于 Miner 线性累积损伤理论推导的疲劳寿命计算公式。分析了不同方向随机振动、正弦振动激励作用下的钢丝网套 补偿器疲劳损伤情况。结果显示, 在随机振动工况和正弦振动工况下, 钢丝网套补偿器在横向 $(X$ 方向)上的损伤均最大, 且 在设计使用寿命 $(1 \mathrm{~min})$ 以内均未发生疲劳破坏; 钢丝网套补偿器的薄弱环节分布于补偿器个别波峰以及管接头固定端附近; 正弦振动工况下损伤水平小于随机振动工况下损伤水平。
\end{abstract}

关键词: 补偿器; 波纹管; 疲劳; 随机振动; 正弦振动

中图分类号: $0324 ; \mathrm{TG} 405$

\section{Vibration Fatigue Damage Analysis of Compensator Reinforced by Wire Cloth with Preload}

\author{
YANG Yan ${ }^{1}$ HE Qilin ${ }^{1}$ ZHU Haiyang ${ }^{2}$ SI Huiliu ${ }^{1}$ WANG Qi ${ }^{2}$ REN Mingfa ${ }^{2}$
}

(1. Beijing Institute of Astronautical Systems Engineering, Beijing 100076;

2. Department Engineering Mechanics, Dalian University of Technology, Dalian 116024)

\begin{abstract}
The fatigue analysis of the compensator reinforced by wire cloth under random vibration conditions and sinusoidal vibration conditions based on the finite element analysis software ABAQUS is carried out. Firstly, the beam element is used to simulate the wire cloth, and different interaction conditions between wire cloth and bellow are set for different load conditions, so the model for random vibration and sinusoidal vibration conditions can be established. The reaction test and modal test are carried out to verify the model. Based on the model, the Dirlik method and fatigue life formula based on Miner linear cumulative damage theory are used to calculate the fatigue damage of steel wire mesh compensator under random vibration and sinusoidal vibration excitation in different directions. The results show that under random vibration conditions and sinusoidal vibration conditions, the compensator has the largest damage level in the horizontal direction ( $X$-direction), and( no fatigue damage occurs within design life ( $1 \mathrm{~min})$; weak points of the compensator are located near the individual peaks of the compensator and the fixed point of the pipe joint; the damage level under sinusoidal vibration conditions is less than the damage level under random vibration conditions.
\end{abstract}

Key words: compensator; bellow; fatigue; random vibration; sinusoidal vibration

\section{0 前言}

补偿器是不同管路之间的连接装置, 可对于管 路系统在安装与工作中产生的装配误差和相对位移

20200820 收到初稿, 20201106 收到修改稿
进行一定的调整，并吸收管路在工作过程中产生的 冲击和振动能量, 能起到降噪和减震的作用, 从而 保证管路连接系统的安全性与可靠性。

目前在工程中使用的补偿器通常为外部带有钢 丝网套的波纹管。波纹管较柔软且密封性好, 是管 路结构中实现 “补偿” 功能的主要部位。在工程实 际中, 波纹管经常在承受较多循环次数的变载荷和 
较大位移的条件下工作, 其疲劳破坏将会使系统失 效。因此, 补偿器的抗疲劳性能对其工程应用具有 重要意义 $义^{[1-3]}$ 。振动疲劳是管路结构使用寿命的一个 重要影响因素。补偿器振动疲劳的传统研究方法是 试验研究, 通过对补偿器开展振动疲劳试验, 对其 进行考核验证, 以得到其疲劳性能。但是该方法不 仅成本高、周期长, 而且难以对全域动态疲劳寿命 进行分析和估测 ${ }^{[4]}$ 。随着计算动力学以及随机理论 研究的发展, 线性结构在振动载荷作用下结构疲劳 寿命的数值计算方法已逐渐成熟。

振动疲劳分析方法主要包括时域法和频域法。 时域法是首先对响应随机过程进行时域模拟, 得到 应力响应时间历程, 然后采用循环计数法进行处理, 得到应力幅值信息, 再根据材料的疲劳寿命曲线和 疲劳累积损伤理论进行疲劳寿命估算 ${ }^{[5]}$ 。用时域法 处理周期性的响应较为直观和方便。但疲劳载荷形 式变为随机载荷时, 载荷变得更为复杂, 若用时域 法处理整个随机加载历程, 则需要大量的随机信号 样本, 计算量较大。频域法是在频域内用功率谱密 度函数(Power spectral density, PSD)的谱参数描述应 力响应, PSD 描述平稳过程随频率变化的特性, 是 随机过程在频域内的重要数值特征量, 表示的是随 机过程的能量分布 ${ }^{[5]}$ 。频域法是结合材料的疲劳寿 命曲线和疲劳累积损伤理论进行寿命估算, 由于该 方法不需进行循环计数, 计算简单, 受到了学术界 和工程界的重视 ${ }^{[6-7]}$ 。目前在国内外对振动疲劳寿命 估算的频域法已经开展了较多研究。DIRLIK ${ }^{[8]}$ 用 Monte Carlo 法进行全面的计算机模拟, 研究了七十 种不同形状的 PSD 函数, 最后用一个指数分布和两 个 Rayleigh 分布近似雨流循环幅值概率密度函数, 与利用窄带分布法得到的结果及其修正公式相比, 计算更为准确; 彭丽华 ${ }^{[9]}$ 提出了一种基于预留计数 法的疲劳损伤频域计算方法, 分析获得的热点应力 功率谱密度生成应力随时间的分布, 进行累积损伤 计算, 其结果具有可靠性; 智东平 ${ }^{[10]}$ 提出了基于有 限元动力学计算和疲劳寿命估计理论相结合的振动 疲劳寿命分析方法, 先对结构进行模态分析, 再进 行频响分析以及随机振动分析, 并结合 Matlab, 计 算随机振动疲劳寿命, 并验证了该方法的适用性; BENASCIUTTI 等 ${ }^{[11}$ 提出了一种新的疲劳寿命频域 分析方法, 指出窄带分布法的预测结果在宽带随机 过程下偏于保守, Dirlik 方法的预测结果较为准确。

建立准确的有限元分析模型并根据补偿器的材 料性能、工况等因素选用合适的疲劳寿命分析方法 是采用数值方法进行补偿器振动疲劳寿命分析的重
点 ${ }^{[2-15]}$ 。近年来, 赵永亮 ${ }^{[16]}$ 针对运载器多层波纹管 路建立了管路低周疲劳设计理论模型, 提出了数值 分析方法以及低周疲劳寿命预测方法, 为波纹管路 的设计提供了理论基础; 田坤等 ${ }^{[17]}$ 使用 MSC Fatigue 疲劳寿命分析软件分析了 $\mathrm{U}$ 形波纹管的高 周疲劳寿命, 并与传统经验公式计算所得的结果进 行对比, 二者吻合较好, 验证了该方法预测波纹管 疲劳寿命的可行性; 于长波等 ${ }^{[18]}$ 借助 ANSYS 有限 元分析软件, 利用多层波纹管的局部应力应变状态 对波纹管的疲劳寿命进行了预测, 为其疲劳设计提 供了依据; 黄乃宁等 ${ }^{[19]}$ 以应变寿命法分析了金属波 纹管在压力、位移载荷作用下的疲劳寿命, 并通过 疲劳试验验证了软件分析的准确程度, 结果表明利 用有限元分析疲劳寿命的结果准确性较好; 薛立鹏 等 ${ }^{[20]}$ 推导了基于 Miner 累积损伤理论下结构的正 弦、定频、随机振动的疲劳损伤计算公式, 并利用 有限元软件计算了管路结构在相应激励下的疲劳损 伤, 其结果与试验结果吻合良好。以上研究大多是 针对无钢丝网套的波纹管的疲劳寿命分析进行的研 究, 但是针对带钢丝网套的波纹管补偿器的振动疲 劳寿命分析的研究, 特别是考虑其带预载情况下随 机振动工况和正弦振动工况的疲劳寿命分析的研究 较少。

本文使用 ABAQUS 有限元分析软件, 考虑带 钢丝网套波纹管补偿器的材料非线性, 几何非线性 以及钢丝网套与波纹管之间的高度非线性相互作用 关系等问题, 建立带钢丝网套波纹管补偿器有限元 分析模型, 对带有预载的钢丝网套补偿器进行随机 振动、正弦振动分析, 并开展了带预载的钢丝网套 补偿器在随机振动工况、正弦振动工况下的瘦劳寿 命预测, 为工程研制提供预示参考。

\section{1 疲劳分析模型建立}

\section{1 有限元模型建立}

在对钢丝网套补偿器进行疲劳寿命分析之前, 需要先进行静力分析和模态分析以获得结构应力应 变以及振动响应, 从而可以获得应力响应谱以预测 结构振动疲劳寿命。为保证动力学分析的准确性, 需要建立能够正确反映钢丝网套补偿器在实际工况 中的有限元模型。常见的钢丝网套建立方法包括等 效刚度模型以及等效梁模型 ${ }^{[21]}$ 。其中, 等效刚度模 型将网套均匀化为一个光滑的筒壳, 建模简单; 等 效梁模型是采用梁单元来模拟网套上的钢丝, 并通 过交叉点自由度耦合模拟实际服役过程中网套编织 
角的变化 ${ }^{[22]}$ 。为比较上述两种模型计算的准确性, 分别建立了等效刚度模型和等效梁模型, 对其施加 轴向拉伸载荷, 并与试验结果对比, 得到载荷-位移 曲线如图 1 所示。

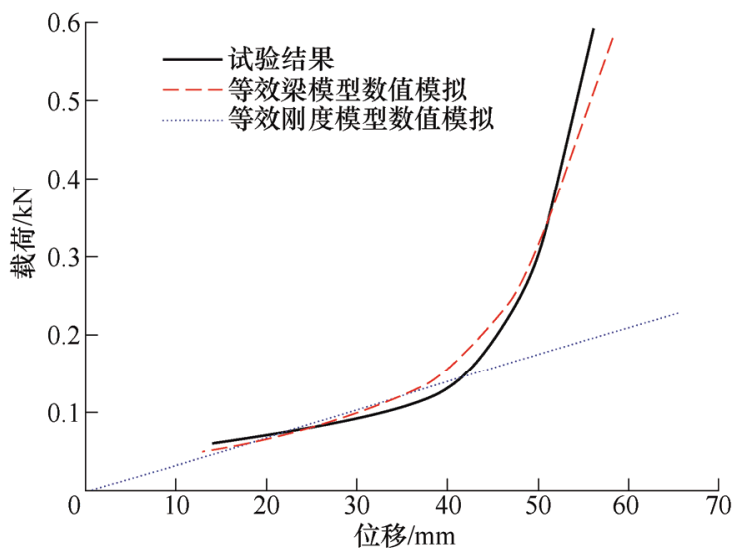

图 1 试验与数值模拟载荷-位移曲线比较

可见, 随着位移增大, 钢丝网套受拉伸载荷作 用, 钢丝间编织角减小, 钢丝网套的刚度逐渐增大, 从而载荷-位移曲线斜率逐渐增大。等效梁模型能够 较好反映这一现象, 而等效刚度模型由于其刚度不 变, 载荷-位移曲线为线性。因此, 本文采用等效梁 模型进行钢丝网套有限元建模。具体建模方法如下:

首先建立互相交织的螺旋线, 再对其赋予梁截 面属性来模拟钢丝网套上的钢丝锭。梁截面宽度设 置为每根钢丝直径之和, 在静力分析中, 为保证计 算的收玫性, 钢丝单元的梁截面属性使用高度等效, 这样即可保证网套与波纹管在几何上刚好接触; 在 动力分析中, 使用面积等效原则, 即梁模型高度按 照钢丝总面积获得。过梁截面中心的法线与螺旋线 切向重合, 梁截面高度方向与螺旋线曲率半径方向 平行。两个方向的网套交叉节点之间设置耦合柱坐 标系下的三个平动自由度, 即使两个方向的网套在 交叉点处能够有相同的平动位移。静力分析中, 网 套与波纹管之间设为接触关系, 动力分析中, 网套 与波纹管之间耦合三个平动自由度, 波纹管波峰位 置划分偶数个网格, 使波纹管峰上存在节点, 实现 耦合设置。

\section{2 随机振动疲劳分析方法}

为估算疲劳寿命, 需要建立疲劳寿命与外载荷 之间的关系。描述外加应力 $S$ 与疲劳寿命 $N$ 之间关 系的曲线称为 $S-N$ 曲线。 $S-N$ 曲线的最常用形式为 幂函数型, 其表达式如下

$$
S^{\alpha} N=C
$$

式中, $C$ 和 $\alpha$ 为材料常数。

因为本文需要实现带预载情况下的疲劳寿命分
析, 故需要考虑初始预应力对疲劳的影响。式(1)可 改写为

$$
S^{\alpha} \cdot N \cdot\left(\frac{\sigma_{b}}{\sigma_{b}-\sigma_{z}}\right)^{\alpha}=C
$$

式中, $\sigma_{b}$ 为材料的强度极限; $\sigma_{z}$ 为初始预应力。

应力概率密度函数在随机载荷作用下，可改写 为 $p(S)$, 式(2)可表示为

$$
\int_{0}^{+\infty} S^{\alpha} \cdot P(S) \mathrm{d} S \cdot N \cdot\left(\frac{\sigma_{b}}{\sigma_{b}-\sigma_{z}}\right)^{\alpha}=C
$$

Dirlik 假定雨流循环幅值概率密度函数是一个 指数分布与两个 Raileigh 分布, 其表达式为

$$
N(s)=E[p] \cdot T \cdot p(s)
$$

式中, $N(s)$ 为发生在时间 $T$ 内应力为 $s$ 的循环次数

$$
\begin{gathered}
p(S)=\frac{\frac{D_{1}}{Q} \exp \left(\frac{-Z}{Q}\right)+\frac{D_{2} Z}{R^{2}} \exp \left(\frac{-z^{2}}{2 R^{2}}\right)+D_{3} Z \exp \left(\frac{-Z^{2}}{2}\right)}{2 \sqrt{m_{0}}} \\
D_{1}=\frac{2\left(\chi_{m}-\gamma^{2}\right)}{1+\gamma^{2}} \quad D_{2}=\frac{1-\gamma-D_{1}+D_{1}^{2}}{1-R} \\
D_{3}=1-D_{1}-D_{2} \quad Z=\frac{S}{2 \sqrt{m_{0}}} \\
Q=\frac{1.25\left(\gamma-D_{3}-\gamma-D_{2} R\right)}{D_{1}} \quad R=\frac{\gamma-\chi_{m}-D_{1}^{2}}{1-\gamma-D_{1}+D_{1}^{2}} \\
\gamma=\frac{m_{2}}{\sqrt{m_{0} m_{4}}} \quad \chi_{m}=\frac{m_{1}}{m_{0}} \sqrt{\frac{m_{2}}{m_{4}}}
\end{gathered}
$$

则联立可得随机振动疲劳损伤计算公式

$$
D=\int_{0}^{+\infty} \frac{n(s)}{N}=\int_{0}^{+\infty} \frac{S^{\alpha} E[p] T p(s)}{C} \mathrm{~d} s
$$

式中, $E[p]$ 为峰值期望值, 其计算公式

$$
E[p]=\sqrt{\frac{m_{4}}{m_{2}}}
$$

式中, $m_{2}$ 为功率谱密度曲线下的第 2 阶惯性矩; $m_{4}$ 分别为功率谱密度曲线下的第 4 阶惯性矩。

考虑预应力进行修正, 损伤结果如下

$$
\bar{D}=\left(\frac{\sigma_{b}}{\sigma_{b}-\sigma_{z}}\right)^{\alpha} D
$$

\section{3 正弦振动疲劳分析方法}

在 Miner 线性累积损伤理论中，定义疲劳损伤 $D$ 为工作应力下载荷的循环次数 $n$ 与工作应力下材 
料的疲劳寿命 $N$ 的比值

$$
D=\frac{n}{N}
$$

由材料 S-N 曲线可得

$$
N=\frac{C}{S^{\alpha}}
$$

正弦振动过程中, 振动时间、频率和扫描率三 者之间的关系

$$
t=\frac{\ln \left(f / f_{0}\right) / \ln 2}{R}
$$

式中, $R$ 为正弦振动扫描率, 单位为 oct $/ \mathrm{s}$ 。在本文 中, $R=0.4 \mathrm{oct} / \mathrm{s}$ 。

上式等号两侧对 $f$ 求导数, 则有

$$
\frac{\mathrm{d} t}{\mathrm{~d} f}=\left[\frac{\ln (f)-\ln \left(f_{0}\right)}{R \ln 2}\right]=\frac{1}{f R \ln 2}
$$

则损伤计算为

$$
D=\int_{0}^{T} \frac{f S^{\alpha}}{C} \mathrm{~d} t=\int_{f_{1}}^{f_{2}} \frac{S^{\alpha}}{C} \frac{\mathrm{d} f}{R \ln 2}
$$

考虑预应力进行修正, 损伤结果

$$
\bar{D}=\left(\frac{\sigma_{b}}{\sigma_{b}-\sigma_{z}}\right)^{\alpha} D
$$

\section{2 模型验证}

开展带钢丝网套波纹管补偿器的支反力测试试 验以及自振频率测试试验, 并将结果与上述有限元 分析模型对比, 以验证其准确性。

建立钢丝网套波纹管补偿器有限元模型, 如 图 2 所示, 在两端各建立一个参考点, 分别把补偿 器两端自由度耦合至参考点上, 并在一端参考点施 加横向 $20 \mathrm{~mm}$ 位移, 另一端参考点固定, 提取固定 端支反力和支反力矩。

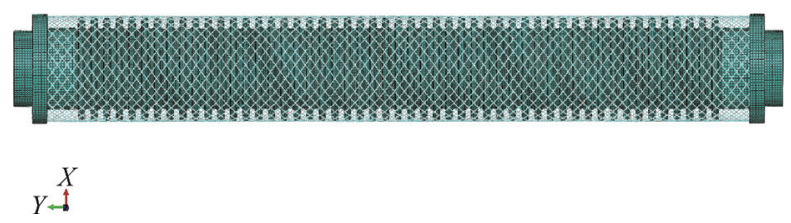

图 2 补偿器有限元模型

本文通过开展钢丝网套波纹管补偿器静力试 验, 测试其支反力, 以验证本文有限元模型静力分
析的准确性。建立了补偿器支反力测试系统, 采用 液压传动器在补偿器一端施加 $20 \mathrm{~mm}$ 的横向位移, 在补偿器另一端连接六维力传感器并将其固定(如 图 3 所示), 采用六维力传感器测量支反力和支反力 矩。使用本文建立的有限元模型对钢丝网套波纹管 补偿器进行静力分析, 对其施加与试验条件相同的 位移载荷和边界条件, 提取固定端支反力和支反力 矩计算结果, 并与试验结果进行对比, 如表 1 所示。 可见, 本文所建立的有限元模型静力工况预测误差 不大于 $12.6 \%$ 。

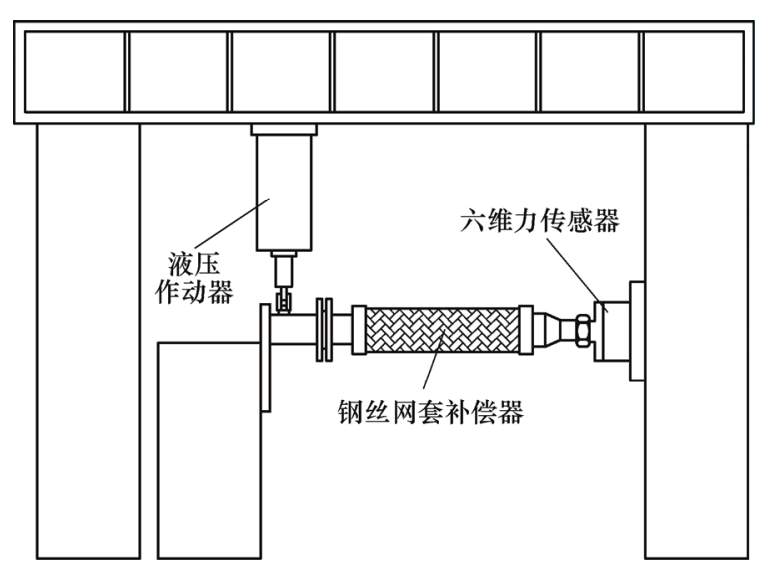

图 3 补偿器支反力测试系统

表 1 支反力计算结果与试验结果对比

\begin{tabular}{ccc}
\hline 比较项 & 支反力/ & 支反力矩 $/(\mathrm{N} \cdot \mathrm{m})$ \\
\hline 有限元计算结果 & 30.6 & 13.2 \\
试验测试结果 & 35 & 15 \\
误差 $(\%)$ & 12.6 & 12.0 \\
\hline
\end{tabular}

随后开展模态测试试验, 以验证本模型动力分 析的准确性。搭建钢丝网套补偿器模态测试系统, 在补偿器的不同位置设置测点, 测点排布如图 4 所 示。在每个测点安装加速度传感器, 将传感器与 LMS 数据采集分析系统连接, 并将补偿器两端固 定, 如图 5 所示。用力锤敲击金属管, 测量振动响 应和激励力, 同时采集力锤激励信号和加速度测点 响应信号, 通过测量激励力和系统的响应输出, 从 而得到系统的频响函数。结合实测频响函数和频响 函数的理论公式, 再利用最小二乘复频域法 $(\text { PolyMax 算法 })^{[23]}$, 得到结构的自振频率。

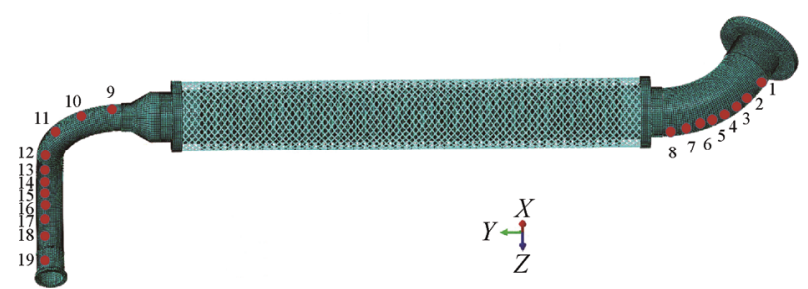

图 4 补偿器有限元模型 


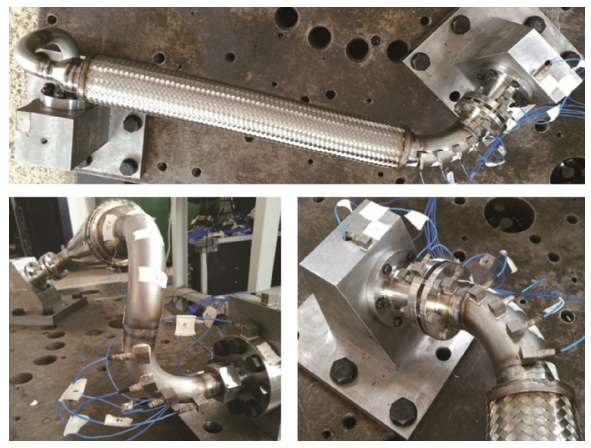

图 5 补偿器模态测试系统

基于本文建立的有限元模型, 使用 Lanczos 法 计算其自振频率, 提取前 4 阶非零频率。表 2 对比 了有限元计算的自振频率结果与试验结果。可见, 本模型前四阶自振频率最大预测误差为 $12.8 \%$ 。综 合上述静力试验与模态测试试验结果, 本文所建立 的模型预测误差约为 $12 \%$, 具有良好的精度, 能够 较好地满足工程需求。

表 2 自振频率计算结果与试验结果对比

\begin{tabular}{ccccc}
\hline 阶数 & 1 & 2 & 3 & 4 \\
\hline 数值结果 $/ \mathrm{Hz}$ & 98.68 & 111.05 & 220.27 & 231.32 \\
试验结果 $/ \mathrm{Hz}$ & 91.3 & 102.8 & 195.2 & 206.3 \\
误差 $(\%)$ & 8.1 & 8.0 & 12.8 & 12.1 \\
\hline
\end{tabular}

\section{3 算例分析}

\section{1 预载工况分析}

本文中, 带钢丝网套补偿器在工作环境下通常 是承受内压 $0.15 \mathrm{MPa}$, 横向位移 $20 \mathrm{~mm}$ 的载荷, 所 以本文按照以上工况，对图 4 所示有限元模型进行 预载工况分析, 补偿器左端固定, 右端沿 $Z$ 轴负方 向移动 $20 \mathrm{~mm}$, 波纹管内受均压 $0.15 \mathrm{MPa}$, 分析结 构静应力响应。上述工况下, 补偿器 Mises 应力云 图如图 6 所示。

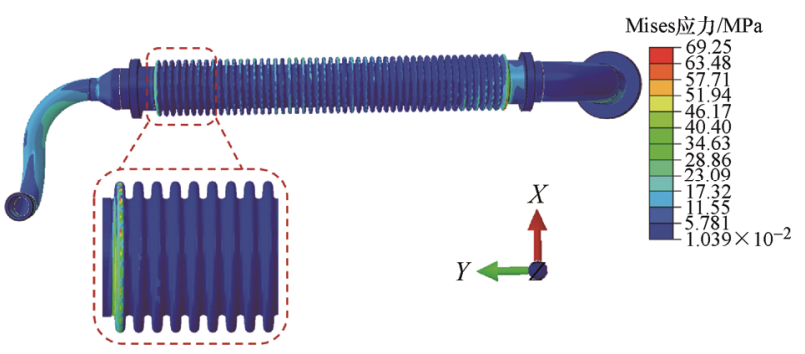

图 6 补偿器 Mises 应力云图

可以发现, 补偿器的最大应力位于波纹管左端 第一个波峰处, 大小 $69.2 \mathrm{MPa}$, 说明波纹管左端第 一个波峰处附近是补偿器承受静载荷时最易发生破 坏的部位。

\section{2 随机振动分析}

保持钢丝网套补偿器的预载不变, 分别分析了 $X, Y, Z$ 三个方向随机振动激励下的响应情况, 激 励如图 7 所示。获得补偿器在上述三种情况下均方 根应力以及补偿器最大均方根应力区域的 PSD 分 布。补偿器和左侧管接头的均方根应力云图如图 8 所示, 提取 Mises 均方根应力最大位置的 PSD 曲线 如图 9 所示。

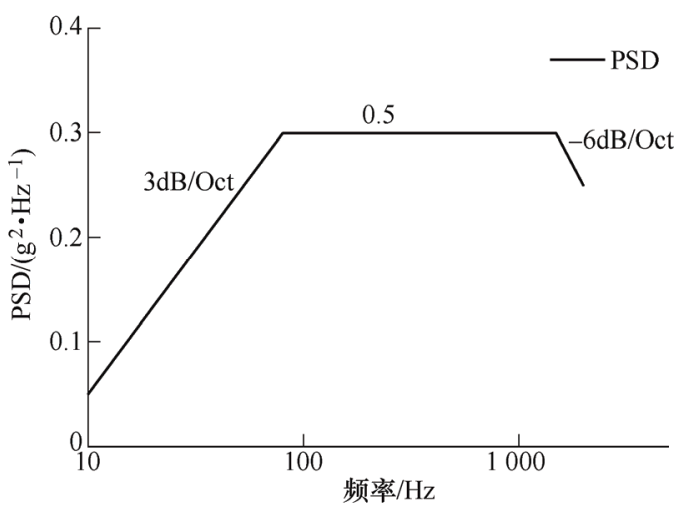

图 7 随机振动激励谱

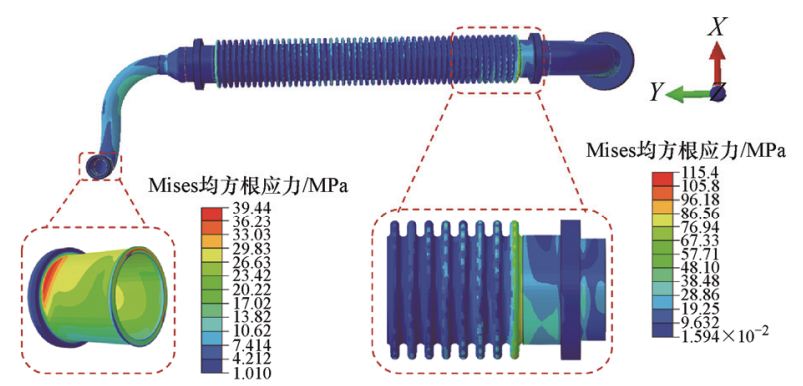

(a) 横向 $(X$ 方向)激励下补偨器、管接头Mises均方根应力云图

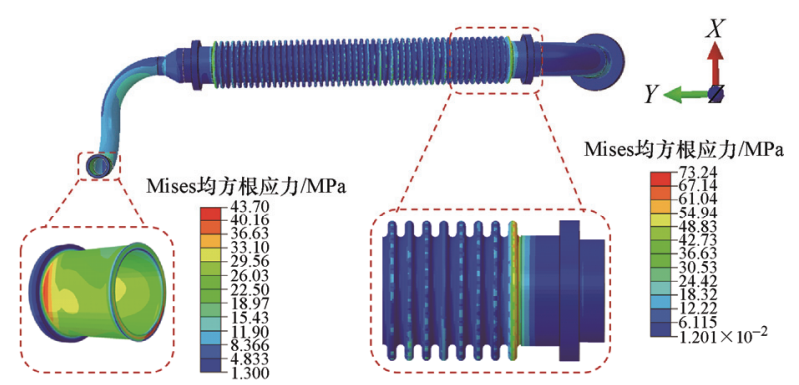

(b) 纵向 $(Y$ 方向)激励下补偿器、管接头Mises均方根应力云图

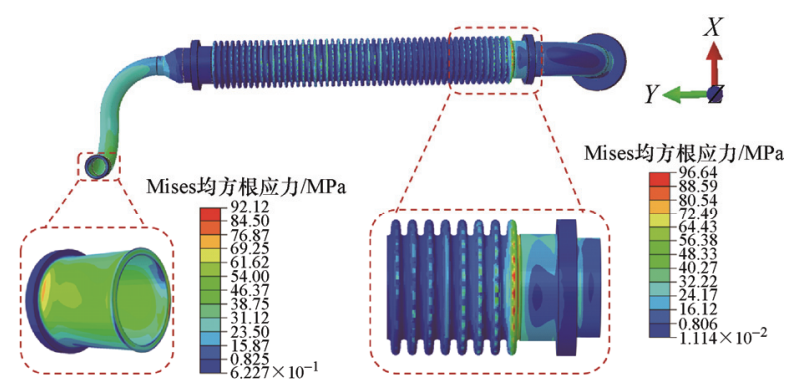

(c) 横向 $(Z$ 方向)激励下补偿器、管接头Mises均方根应力云图

图 8 补偿器、管接头 Mises 均方根应力云图 


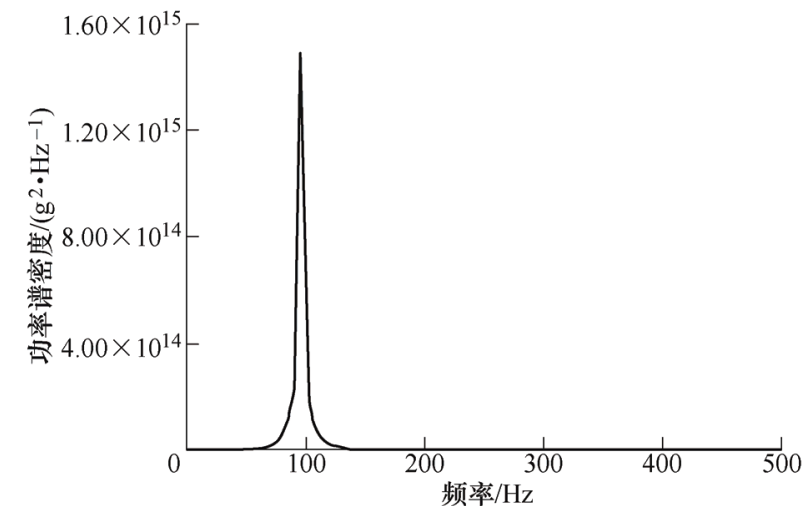

(a) 横向 $(X$ 方向) 激励下补偿器危险点PSD曲线

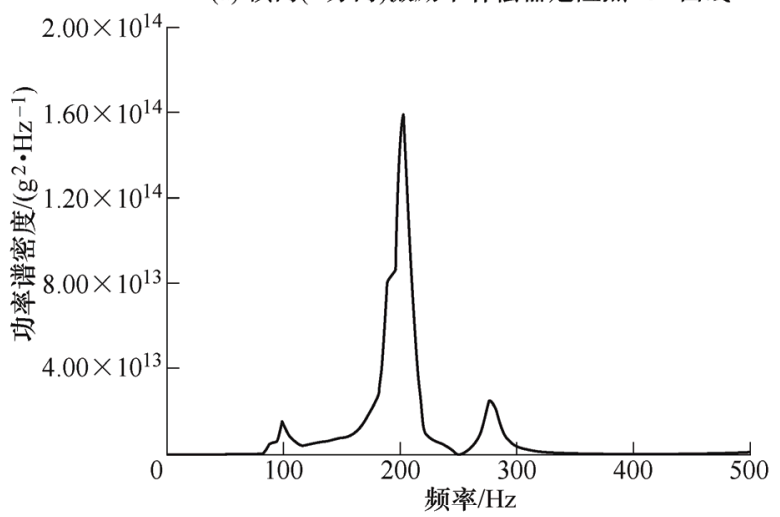

(b) 纵向 $(Y$ 方向) 激励下补偿器危险点PSD曲线

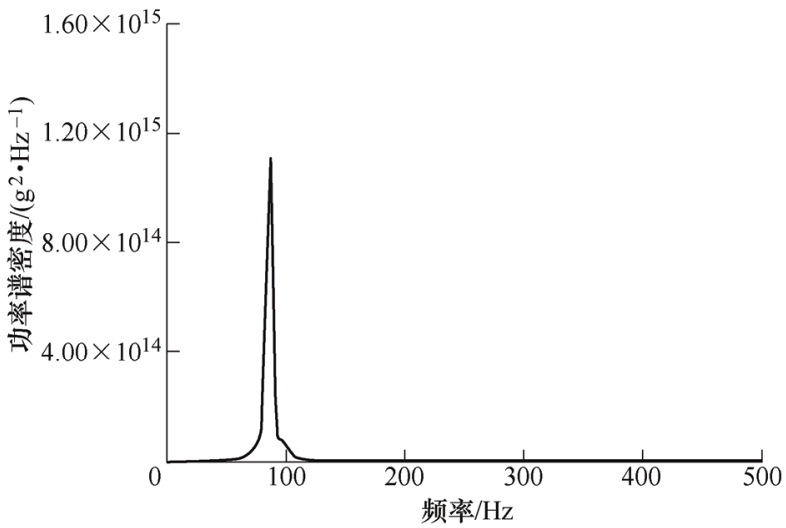

(c) 横向 $(Z$ 方向)激励下补偿器危险点PSD曲线

图 9 补偿器危险点 PSD 曲线

从图 8 , 图 9 可看出, 在横向 $(X$ 方向)随机振动 激励下, 最大 Mises 均方根应力出现在波纹管最右 侧第一个波峰处, 约 $115.4 \mathrm{MPa}$, 对应第 1 阶自振 频率的激励; 在纵向 ( $Y$ 方向)随机振动激励下, 最大 Mises 均方根应力出现在波纹管右侧与接头相连部 位附近, 约 73.2 MPa, 对应第 2 阶自振频率的激励; 在横向 $(Z$ 方向)随机振动激励下, 最大 Mises 均方根 应力出现在波纹管最右侧第一个波峰处, 约 96.6 $\mathrm{MPa}$, 对应第 1 阶自振频率的激励。除此之外, 补 偿器左侧管接头的固定端附近的 Mises 均方根应力 水平较大, 且在 $Z$ 方向随机振动激励下管接头固定 端附近的 Mises 均方根应力水平明显大于横向或纵 向随机振动激励下管接头固定端附近的 Mises 均方
根应力水平, 说明管接头固定端附近也是补偿器随 机振动工况中的一个薄弱环节, 且 $Z$ 方向的随机振 动激励对于管接头固定端附近的 Mises 均方根应力 贡献较大。从图 9 可看出, 补偿器在横向 $(X$ 方向或 $Z$ 方向)上的随机振动响应主要来自于第 1 阶自振频 率激励, 在纵向 $(Y$ 方向)上的随机振动响应主要来自 于第 2 阶自振频率激励。

\section{3 正弦振动分析}

保持钢丝网套补偿器的预载不变, 开展振动扫 频分析, 分别分析了 $X, Y, Z$ 三个方向正弦振动 激励下的响应情况, 计算上述三种情况下补偿器 的等效 Mises 应力, 并提取 Mises 应力最大位置的 频响曲线, 补偿器和左侧管接头的 Mises 应力云图 如图 10 所示、相应的 Mises 应力响应谱如图 11 所示。

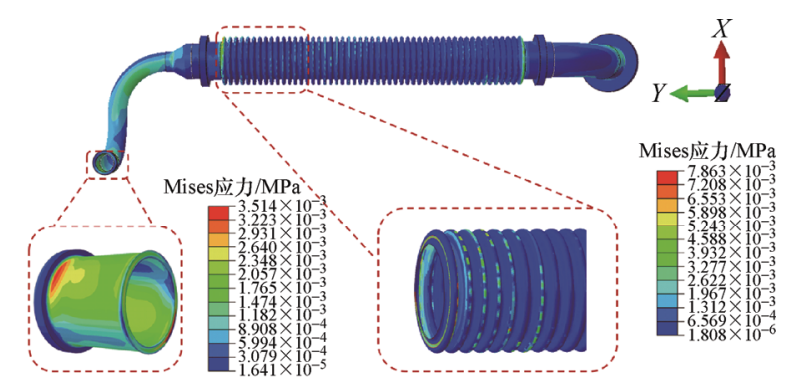

(a) 横向 $(X$ 方向)激励下补偿器、管接头Mises应力云图

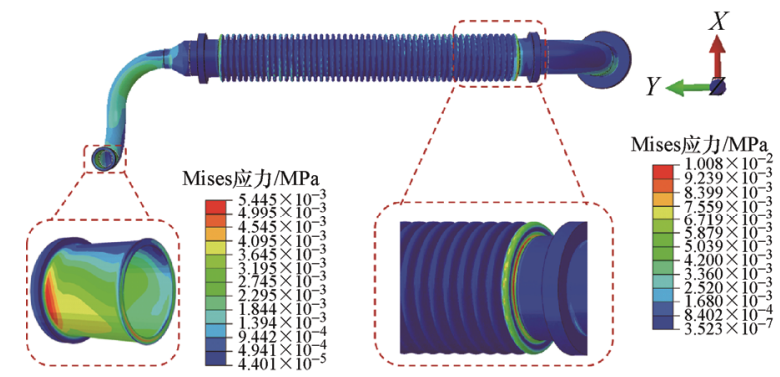

(b) 纵向 $(Y$ 方向)激励下补偿器、管接头Mises应力云图

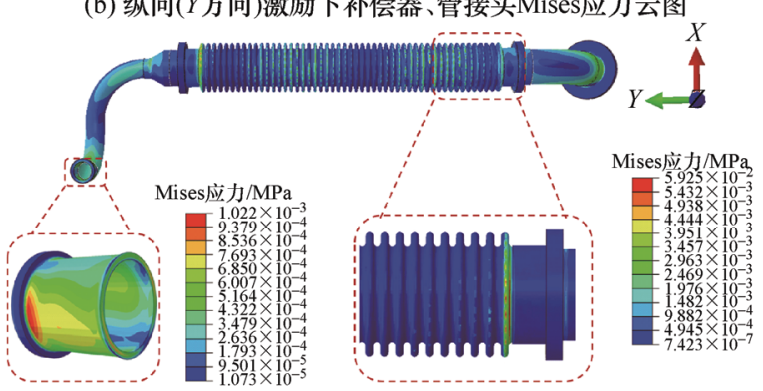

(c) 横向 $(Z$ 方向)激励下补偿器、管接头Mises应力云图

图 10 补偿器、管接头 Mises 应力云图

从图 10 可看出, 横向 $(X$ 方向 $)$ 正弦振动激励下, 补偿器最大 Mises 应力分布在右侧第 5 个波峰处; 纵向 $(Y$ 方向)正弦振动激励下, 补偿器最大 Mises 应 力分布在右侧第 1 个波谷处; 横向 $(Z$ 方向)正弦振动 激励下, 补偿器最大 Mises 应力分布在右侧第 1 个 波峰处。除此之外, 补偿器左侧管接头的固定端附 
近的 Mises 应力水平较大, 且在纵向 $(Y$ 方向)正弦振 动激励下管接头固定端附近的 Mises 应力水平较 大, 说明管接头固定端附近也是补偿器正弦振动工 况中的一个薄弱环节, 且纵向 ( $Y$ 方向) 的正弦振动激 励对于管接头固定端附近的 Mises 应力贡献较大。 从图 11 可看出, 补偿器在横向 $(X$ 方向或 $Z$ 方向)上 的正弦振动响应主要来自于第 1 阶自振频率的激 励; 在纵向 $(Y$ 方向)上的正弦振动响应主要来自于第 2 阶自振频率的激励。

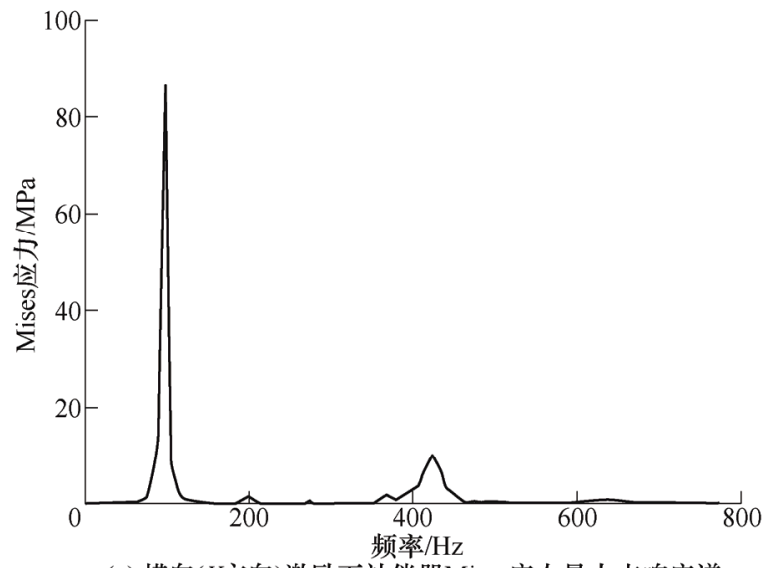

(a) 横向 $(X$ 方向)激励下补偿器Mises应力最大点响应谱

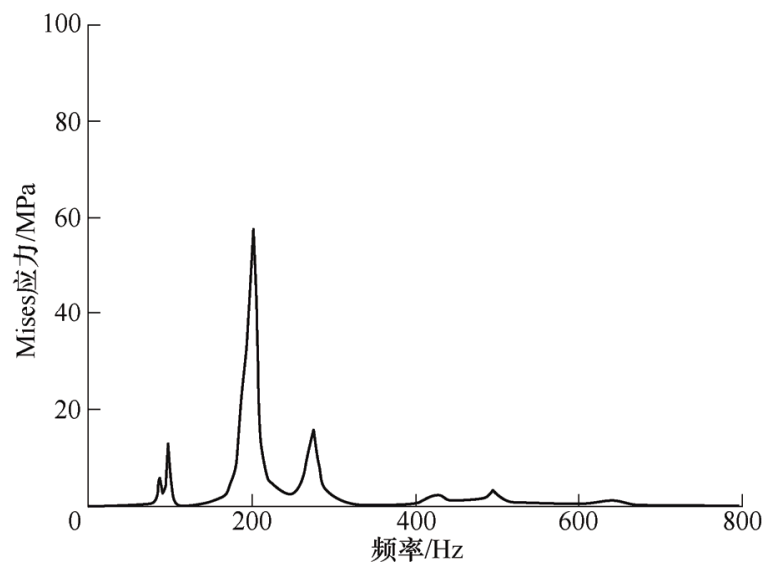

(b) 纵向 $(Y$ 方向)激励下补偿器Mises应力最大点响应谱

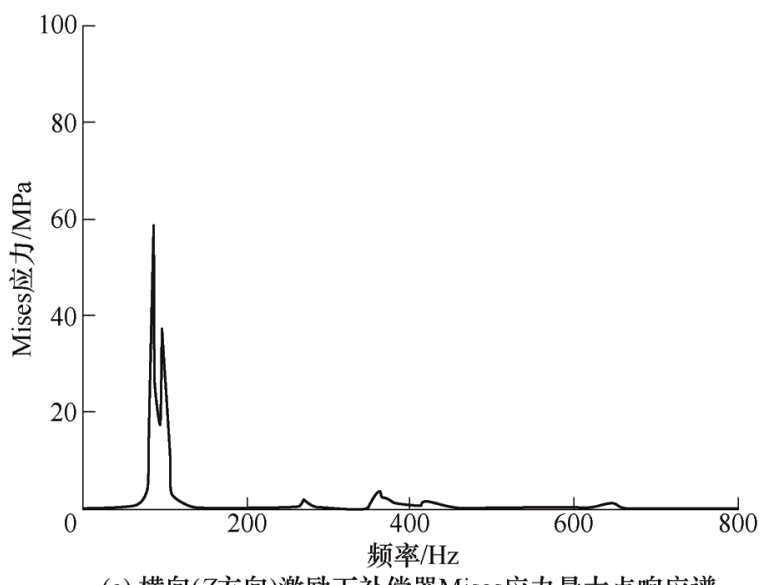

(c) 横向 (Z方向)激励下补偿器Mises应力最大点响应谱

图 11 Mises 应力最大点应力响应谱

\section{4 疲劳损伤分析}

本文所用钢丝网套补偿器的材料为 $0 \mathrm{Cr} 18 \mathrm{Ni}$,
其 $S-N$ 曲线为 $S^{5.14} N=10^{19}$ [5]。本文研究的钢丝网 套补偿器工作时间为 $1 \mathrm{~min}$, 故随机振动和正弦振动 的试验时间选取为 $1 \mathrm{~min}$ 。

对于考虑预载影响的随机振动疲劳寿命分析, 先求出分别在 $X 、 Y 、 Z$ 三个方向随机载荷激励时波 纹管最大均方根应力处应力谱密度 $G(f)$, 采用 Dirlik 疲劳寿命分析方法, 应用式(4) (8), 计算得到的波 纹管疲劳损伤结果如表 3 所示。

表 3 随机振动疲劳分析结果

\begin{tabular}{cccc}
\hline 随机振动激励方向 & $X$ & $Y$ & $Z$ \\
\hline$D$ & 0.0570 & 0.0071 & 0.0176 \\
\hline
\end{tabular}

对于考虑预载影响的正弦振动疲劳寿命分析, 先求出分别在 $X 、 Y 、 Z$ 三个方向正弦载荷激励时波 纹管最大 Mises 应力处 Mises 应力谱密度 $G(f)$, 应 用式(9) (14), 计算得到的波纹管疲劳损伤结果如 表 4 所示。

表 4 正弦振动疲劳分析结果

\begin{tabular}{cccc}
\hline 正弦振动激励方向 & $X$ & $Y$ & $Z$ \\
\hline$D$ & $9.47 \times 10^{-9}$ & $2.79 \times 10^{-9}$ & $8.82 \times 10^{-10}$ \\
\hline
\end{tabular}

可见,波纹管在横向 $(X$ 方向)随机振动激励下的 损伤和正弦振动激励下的损伤均最大, 其原因为: 波纹管补偿器在横向 $(X$ 方向)没有约束, 故在该方向 激励下补偿器易产生较大位移和变形, 并进一步引 起补偿器两端的接头处产生弯曲载荷, 导致接头倒 角处具有较高的应力水平。此外, 由模态分析结果 来看, 导管的前 6 阶振型均在横向产生较大位移, 也验证了上述分析。根据 Miner 线性损伤理论, 当 $D<1$ 时, 可以判定结构不会发生疲劳破坏, 从计算 结果看, 无论随机振动和正弦振动的激励方向如何 变化, 波纹管的 $D$ 均小于 1 , 因此带预载的波纹管 在随机振动工况和正弦振动工况下均不会发生疲劳 破坏; 观察表 3 和表 4, 发现钢丝网套补偿器在正 弦振动工况下损伤的总体水平小于随机振动工况下 损伤的总体水平，且二者相差 6 到 8 个数量级。

本文还开展了带钢丝网套补偿器随机振动下的 疲劳试验, 随机振动加载时间为 $30 \mathrm{~min}$ 。试验后钢 丝网套补偿器结构完好, 进行气密性检查, 检查结 果合格，可见本文预测结果偏于安全。

\section{4 结论}

本文建立了钢丝网套补偿器的分析模型, 并实 现了钢丝网套补偿器在随机振动工况和正弦振动工 况下的疲劳损伤分析。 
(1) 在随机振动工况和正弦振动工况下, 钢丝 网套补偿器在横向 $(X$ 方向)上的损伤均最大, 故横向 ( $X$ 方向)为补偿器的最危险方向。

(2) 补偿器中波纹管的个别波峰以及管接头固 定端附近的均方根应力、Mises 应力水平较大, 是 补偿器的薄弱环节。

(3) 在随机振动工况和正弦振动工况下, 经过 设计使用寿命 $(1 \mathrm{~min})$ 的载荷作用, 补偿器在任何方 向上均不会发生疲劳破坏。

(4) 钢丝网套补偿器在正弦振动工况下疲劳损 伤的总体水平明显小于随机振动工况下疲劳损伤的 总体水平。

\section{参 考 文 献}

[1] 徐中华，买买提明・艾尼，程伟. $\mathrm{S}$ 型焊接金属波纹管 疲劳寿命的有限元分析[J]. 压力容器, 2009, 26(2): 21-25.

XU Zhonghua, MAIMAITIMING Aini, CHENG Wei. Finite element analysis to fatigue life of the S-shape bellows[J]. Pressure Vessel Technology, 2009, 26(2): 21-25.

[2] 屈彩虹, 王心丰，岳林. 基于 ANSYS 的金属软管网套 的有限元研究[J]. 机械工程师, 2006(9): 140-142.

QU Caihong, WANG Xinfeng, YUE Lin. Finite element analysis of metallic hose net on ANSYS[J]. Mechanical Engineer, 2006(9): 140-142.

[3] 盛冬平, 朱如鹏, 王心丰, 等. 基于 ANSYS 的金属软 管的瞬态动力学分析 [J]. 压力容器, 2008, 25(1): 14-17. SHENG Dongping, ZHU Rupeng, WANG Xinfeng, et al. Transient dynamic finite element analysis of metallic hose based on ANSYS[J]. Pressure Vessel Technology, 2008, 25(1): 14-17.

[4] 方红荣, 薛立鹏, 李朝晖. 基于有限元法的运载火箭管 路随机振动疲劳寿命分析 [J]. 导弹与航天运载技术, 2017, 354(4): 107-110.

FANG Hongrong, XUE Lipeng, LI Zhaohui. Research on simulation of launch vehicle pipeline structure's random vibration fatigue lifetime based on finite element method[J]. Missles and Space Vehicles, 2017，354(4): 107-110.

[5] 王明珠. 结构振动疲劳寿命分析方法研究[D]. 南京: 南 京航空航天大学, 2009.

WANG Mingzhu. Research on life analysis method for structure vibration fatigue[D]. Nanjing : Nanjing University of Aeronautics and Astronautics, 2009.
[6] 栗玉领, 徐胜利, 杨树桦, 等. 非典型气动荷载下压缩 机叶轮疲劳强度分析 $[\mathrm{J}]$. 机械工程学报, 2015, 51(9): 82-89.

LI Yuling, XU Shengli, YANG Shuhua, et al. Fatigue strength analysis of compressor impeller under non-typical aerodynamic load[J]. Journal of Mechanical Engineering, 2015, 51(9): 82-89.

[7] 张子璠, 杨广雪, 李强, 等. 地铁转向架构架台架试验 载荷谱编制方法 $[\mathrm{J}]$. 机械工程学报，2020，56(1): $1-9$.

ZHANG Zifan, YANG Guangxue, LI Qiang, et al. Compilation method of load spectrum for the bench test of metro bogie frame[J]. Journal of Mechanical Engineering, 2020, 56(1): 1-9.

[8] DIRLIK.T. Application of computers in fatigue analysis[J]. University of Warwick, 1985.

[9] 彭丽华. 考虑雨流计数的频域疲劳计算方法 [D]. 武汉: 武汉理工大学, 2018.

PENG Lihua. A new frequency domain fatigue damage computational method considering rain-flow counting[D]. Wuhan: Wuhan University of Technology, 2018.

[10] 智东平. 航天结构振动疲劳寿命估计方法研究[D]. 南 京: 南京航空航天大学, 2017.

ZHI Dongping. Research on the fatigue life of the astronautic structures under random vibration environment[D]. Nanjing : Nanjing University of Aeronautics and Astronautics, 2009.

[11] BENASCIUTTI D, TOVO R. Comparison of spectral methods for fatigue analysis of broad-band Gaussian random processes $[\mathrm{J}]$. Probabilistic Engineering Mechanics, 2006, 21(4): 287-299.

[12] 赵礼辉, 郑松林, 冯金芝. 基于低载强化特性的疲劳寿 命估计方法 [J]. 机械工程学报, 2013，49(8): 115-122. ZHAO Lihui, ZHENG Songlin, FENG Jinzhi. Fatigue life prediction based on simplified low-amplitude-load strengthening model[J]. Journal of Mechanical Engineering, 2013, 49(8): 115-122.

[13] 欧阳芙, 娄路亮, 方红荣, 等. 随机振动下小导管的疲 劳分析 [J]. 强度与环境，2011，38(5)：59-63.

OUYANG Fu, LOU Luliang, FANG Hongrong, et al. Random vibration fatigue analysis of small pipe[J]. Structure \&Environmental Engineering, 2011, 38(5): 59-63.

[14] 刘德昆, 李强, 王曦, 等. 动车组轴箱轴承基于实测载 荷的寿命预测方法 [J]. 机械工程学报, 2016， 52(22): 
45-54.

LIU Dekun, LI Qiang, WANG Xi, et al. Life prediction method for EMU axle box bearings based on actual measured loadings[J]. Journal of Mechanical Engineering, 2016, 52(22): 45-54.

[15] 周金宇, 韩文钦, 邱睿, 等. 结构系统疲劳失效相关机 理与可靠性模型 $[\mathrm{J}]$. 机械工程学报, 2018, 54(16): 220-226.

ZHOU Jinyu, HAN Wenqin, QIU Rui, et al. Dependence mechanism of fatigue failure and reliability model for structure systems[J]. Journal of Mechanical Engineering, 2018, 54(16): 220-226.

[16] 赵永亮. 运载器多层波纹管路的强度和疲劳寿命分析[D]. 大连: 大连理工大学, 2015.

ZHAO Yongliang. Strength and fatigue life analysis of multilayer bellows for aircraft[D]. Dalian : Dalian University of Technology, 2015.

[17] 田坤, 竺长安. U 形波纹管疲劳寿命研究[J]. 传感器与 微系统, 2011, 30(2): 17-19.

TIAN Kun, ZHU Changan. Study on fatigue life of U-shaped sinu flotube[J]. Transducer and Microsystem Technologies), 2011, 30(2): 17-19.

[18] 于长波, 王建军, 李楚林, 等. 多层 U 形波纹管的疲 劳寿命有限元分析[J]. 压力容器, 2008, 25(2): 23-27. YU Changbo, WANG Jianjun, LI Chulin, et al. Finite element analysis to multilayer U-shaped bellows fatigue life[J]. Pressure Vessel Technology, 2008, 25(2): 23-27.

[19] 黄乃宁, 宋林红, 魏国俭, 等. 阀门用金属波纹管疲劳 寿命的有限元分析[J]. 阀门, 2008, 12(5): 30-34. HUANG Naining, SONG Linhong, WEI Guojian, et al. Fatigue life of finite element analysis metal bellows for
valves[J]. Valves, 2008, 12(5): 30-34.

[20] 薛立鹏, 李文斌, 司群英, 等. 运载火箭结构振动疲劳 损伤的工程分析方法 [J]. 导弹与航天运载技术, 2019, 367(02): 39-42.

XUE Lipeng, LI Wenbin, SI Qunying, et al. Engineering analysis on launch vehicle structural vibration fatigue damage[J]. Missles and Space Vehicles, 2019, 367(02): $39-42$.

[21] 张程. 钢丝网套补偿器有限元分析[D]. 大连: 大连理工 大学, 2010.

ZHANG Cheng. Finite element analysis for compensator reinforced by wire cloth[D]. Dalian: Dalian University of Technology, 2010.

[22] 马军, 葛世荣, 张德坤. 钢丝绳股内钢丝的载荷分布 $[\mathrm{J}]$. 机械工程学报, 2009, 45(4): 259-264.

MA Jun, GE Shirong, ZHANG Dekun. Load distribution on the unit of the wire rope strand[J]. Journal of Mechanical Engineering, 2009，45(4): 259-264.

[23] 张涛, 李强, 王曦, 等. 高速列车齿轮箱箱体在位模态 识别 $[\mathrm{J}]$. 机械工程学报, 2018, 54(12): 31-41.

ZHANG Tao, LI Qiang, WANG Xi, et al. Modal identification of gearbox housing of high speed train under assembly condition[J]. Journal of Mechanical Engineering, 2018, 54(12): 31-41.

作者简介: 杨燕, 女, 1981 年出生, 硕士, 高级工程师。主要研究方向 为管路及压力容器的设计。

E-mail: 28178311@qq.com

任明法(通信作者), 男, 1974 年出生, 博士, 教授, 博士研究生导师。 主要研究方向为复合材料结构力学。

E-mail: renmf@dlut.edu.cn

王琦, 男, 1993 年出生, 博士研究生, 主要研究方向为复合材料工艺力 学分析。

E-mail: 499455885@mail.dlut.edu.cn 